Ann. Sci. forest., $\mathbf{1 9 7 6 ,} 33$ (3), I39-I50.

\title{
PREMIÈRE CONTRIBUTION A L'ÉCONOMIE DE LA FERTILISATION DU PIN MARITIME ADULTE DANS LES LANDES DE GASCOGNE*
}

\author{
G. BUTTOUD \\ Laboratoire d'Économie forestière, C. N. R. F., \\ 14, vue Girardet, \\ 54042 Nancy Cedex
}

RÉSUMÉ

Les premiers résultats d'essais conduits par l'I.N.R.A., permettent de penser que l'apport d'engrais aux pins adultes des Landes est une opération intéressante dont il convient d'approfondir l'étude.

Une fertilisation de type PK, apportée I $5_{5}$ ans environ avant la date de récolte prévue, permettrait d'obtenir un volume supplémentaire de bois d'au moins $25 \mathrm{~m}^{3}$ par hectare et apporterait un bénéfice net actualisé au taux de 5 p. ıoo de $2500 \mathrm{~F}$. Le taux de rentabilité d'un tel investissement serait alors voisin de I5 p. roo.

Les données actuelles ne permettent pas de prévoir avec une précision suffisante quelle importance pourrait prendre la fertilisation adulte dans les Landes, mais le développement des recherches techniques semble une nécessité impérieuse.

Les recherches conduites depuis une quinzaine d'années sur la fertilisation lors de leur installation des peuplements forestiers ont déjà débouché dans certains cas, et notamment dans les Landes de Gascogne, sur des résultats probants qui ont justifié leur application à grande échelle. La réponse spectaculaire du jeune Pin maritime à l'apport de phosphore, la relative homogénéité des peuplements et du sol, le dynamisme des vulgarisateurs, l'intérêt des industriels papetiers et la capacité des propriétaires forestiers en ont facilité l'application. A tel point que depuis quelques années, on peut estimer que presque toutes les régénérations sont systématiquement fertilisées. Parallèlement l'amélioration des techniques culturales apporte

* Cette étude qui est la traduction économique d'une recherche menée par des spécialistes a été réalisée en exćcution de la Convention passée le ro juillet I973 entre le ministère de l'Agriculture (Service des Forêts) et l'Institut national de la Recherche agronomique (Groupe d'Étude et de Recherche en Économie forestière). 
de constants progrès, et la ligniculture qui s'ébauche espère conduire bientôt à une utilisation quasi totale des ressources du sol pour la production du bois.

Mais jusqu'à présent, mis à part le cas particulier de l'apport d'engrais à de jeunes peuplements déficients établis à partir de graines de provenances mal adaptées, peu d'essais de fertilisation ont été conduits sur les pins adultes, et ceci, semble-ti1, pour trois raisons essentielles :

- d'abord et surtout, l'amélioration des techniques de mise en place des peuplements était suffisamment prioritaire pour mobiliser toutes les énergies ;

-- d'autre part, la longue durée d'action du phosphore a poussé à une application précoce ;

- enfin, la faible réponse du Pin maritime à la fertilisation azotée, la plus utilisée sur les peuplements adultes (notamment en Scandinavie) a découragé chercheurs et praticiens.

Or, on peut se demander s'il ne serait pas intéressant de rechercher aussi par apport d'engrais, un supplément de production à court ou moyen terme ; ce pourrait être un moyen de tirer un meilleur parti des peuplements actuels, notamment ceux qui n'ont jamais été fertilisés.

\section{I. - LES DONNÉES TECHNIQUES E'T ÉCONOMIQUES}

\section{I. - Les données techniques}

I. II. Sources.

Faute de pouvoir se fonder sur les résultats d'un échantillon statistique suffisant d'expériences, force est de partir d'un modèle théorique qui a été construit d'après les conclusions que tire M. Bonneau (Station de Recherches sur les sols forestiers et la fertilisation I.N.R.A./C.N.R.F.) (I972) d'essais conduits sous sa direction. A Marcheprime (Gironde) par exemple, une fertilisation importante, appliquée à des pins de 39 ans :

$-\mathrm{PK}=270 \mathrm{~kg} \mathrm{P}_{2} \mathrm{O}_{5} ; 220 \mathrm{~kg} \mathrm{~K}_{2} \mathrm{O} ; 450 \mathrm{~kg} \mathrm{CaO}$

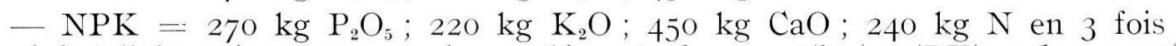
a conduit à l'obtention en Io ans de suppléments de $2, \mathrm{I} \mathrm{m}^{3} / \mathrm{ha} / \mathrm{an}(\mathrm{PK})$ et de $3,5 \mathrm{~m}^{3} /$ ha/an (NPK).

Mais les caractéristiques de cet essai ne sont pas toutes transposables dans la pratique et un certain nombre de données manquent au calcul économique. Il a donc été convenu de "simuler ", en se basant sur les résultats de ces expériences, une fertilisation de pin adulte dans les Landes, qui n'est toutefois qu'une représentation partielle de la réalité.

\section{I2. Le peuplement.}

On considère que le peuplement représenté, qu'on a voulu moyen, appartient à la classe 3 (moyenne de fertilité des peuplements landais) et qu'il est géré de façon traditionnelle comme le décrivent $\mathrm{N}$. Decour'T et B. Lemorne dans les tables de production du Pin maritime (C.N.R.F., I973). Ce peuplement n'a donc auparavant jamais reçu d'engrais. 
I. I3. Modalités d'application.

On a représenté deux essais de fertilisation, l'un avec phosphore et potasse, l'autre avec en plus de l'azote, qu'on appellera par la suite PK et NPK, dans lesquels l'apport est considéré comme une constante; il aurait été intéressant d'étudier l'effet de doses croissantes, mais l'état actuel des recherches ne permet pas une telle approche. L'essai NPK se distingue de PK par deux applications supplémentaires d'azote intervenant $I$ an et 6 ans après 1'apport PK. On suppose que l'épandage s'effectue par tracteur, comme c'est généralement le cas dans les Landes pour la fertilisation au départ, et on admet pour faciliter les calculs qu'il est confié à un entrepreneur. Le tableau suivant résume les principales caractéristiques de cette fertilisation.

TABLEAU I

Quantités apportées et coûts à l'ha

Quantity of fertilizers and costs per ha (details on costs are given later)

\begin{tabular}{|c|c|c|c|c|c|}
\hline \multirow{2}{*}{ Essais } & \multirow{2}{*}{$t$ (années) } & \multicolumn{3}{|c|}{$\begin{array}{l}\text { Quantités apportées } \\
\text { (en kg d'éléments fertilisants) }\end{array}$} & \multirow{2}{*}{$\begin{array}{c}\text { Coûts } \\
\text { (F constants } \\
\text { nov. 1974) }\end{array}$} \\
\hline & & $\mathrm{P}_{2} \mathrm{O}_{5}$ & $\mathrm{~K}_{2} \mathrm{O}$ & N & \\
\hline PK & 0 & 150 & 100 & 一 & 500 \\
\hline NPK & 0 & 150 & 100 & - & 500 \\
\hline 一 & 1 & - & - & 100 & 275 \\
\hline - & 6 & - & - & 100 & 275 \\
\hline
\end{tabular}

(Le détail des coûts présentés est donné plus loin).

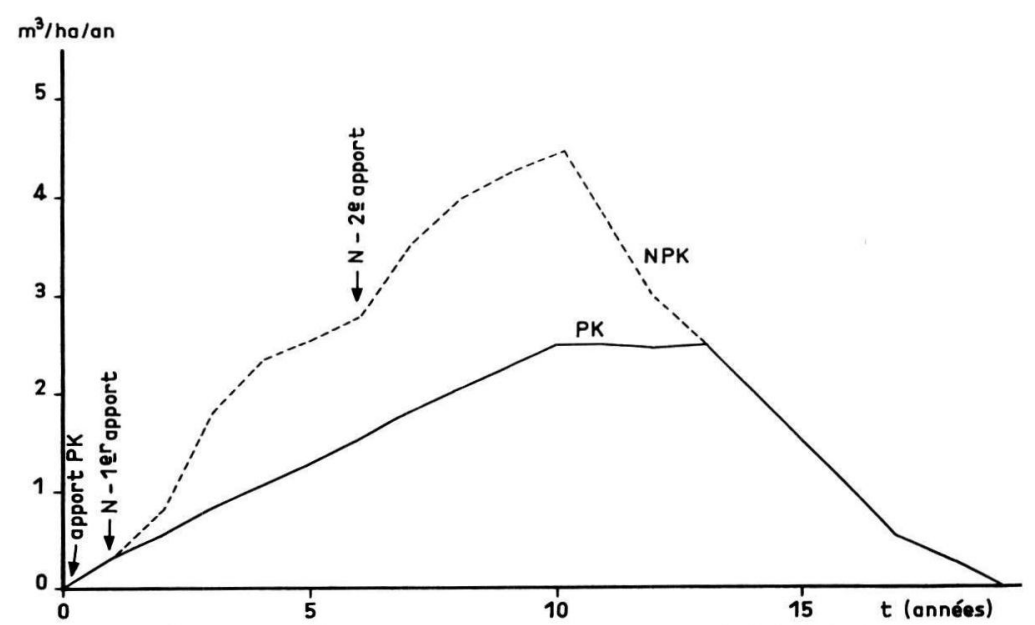

FIG. I. - Suppléments d'accroissement annucls ( $\left.\mathrm{m}^{3} / \mathrm{ha} / \mathrm{an}\right)$

Increase of annual increment $\left(\mathrm{m}^{3} / \mathrm{ha} /\right.$ year $)$ 
I. I4. La réponse physique.
a) Le peuplement principal.

Entre $t=\mathrm{o}$ (date de l'intervention de l'apport phospho-potassique) et $t=\mathrm{I} 9$ (date de cessation hypothétique de l'effet de fertilisation), les suppléments d'accroissement annuel augmentent progressivement pour passer par un maximum avant de décroître et s'annuler. L'effet de l'engrais dure presque 20 ans.

Une telle hypothèse est en fait assez pessimiste ; les pertes de $\mathrm{P}$ par lessivage sont faibles et dans la plupart des cas, la permanence de l'effet de l'apport PK devrait être beaucoup plus grande. D'autre part, il est possible que la même réponse physique puisse être obtenue sans apport potassique donc à prix plus réduit. Toutefois la prudence conduit à retenir dans le calcul une hypothèse de réponse faible qui conduit à 1'obtention des suppléments suivants :

\section{TABLEAU 2}

Suppléments d'accroissement annuel moyens mesurés sur un nombre d'années $\mathrm{n}$ ( $\left.\mathrm{m}^{3} / \mathrm{ha} / \mathrm{an}\right)$

Increase of average annual increment mesured on $n$ years ( $\left.\mathrm{m}^{3} / \mathrm{ha} / \mathrm{year}\right)$

\begin{tabular}{|c|c|c|}
\hline$n$ & PK & XPK \\
\hline 5 & 0,75 & 1,50 \\
\hline 10 & 1,38 & $2,66.5$ \\
\hline 15 & 1,65 & 2,62 \\
\hline 20 & 1,333 & $2,0,5$ \\
\hline
\end{tabular}

La généralisation d'un tel modèle appelle toutefois quelques remarques :

S'il apparaît raisonnable qu'un apport PK permette l'obtention en $I_{5}$ ans d'un supplément d'accroissement annuel moyen d'environ $\mathrm{I}, 5 \mathrm{~m}^{3} / \mathrm{ha}$, il convient d'être plus prudent en ce qui concerne l'azote dont l'effet peut présenter d'une station à une autre des variations non négligeables; la réponse décrite dans le modèle NPK n'est pas assurée partout.

La réponse physique ainsi décrite a été considérée comme fixe et indépendante de 1'âge du peuplement à la date d'apport de l'engrais phospho-potassique. La réalité plus complexe, ferait sans doute état d'une légère diminution de l'effet avec l'âge à la fertilisation, diminution qui peut paraître négligeable par rapport à l'incertitude admise dans le choix des données de réponse.

b) Les éclaircies.

Les tables de production de N. DECOURT et B. LEMOINE (C.N.R.F., I973) font état de la pratique d'éclaircies faibles et nombreuses. L'augmentation $\Delta c$ du volume enlevé lors de ces éclaircies en cas de fertilisation n'est pas négligeable, et a été consi- 
dérée comme proportionnelle à l'accroissement du volume $\Delta v$ du peuplement principal.

$$
\frac{e}{v}=\frac{\Delta e}{\Delta v}
$$

par exemple

$$
\begin{array}{lll}
\text { à } 46 \text { ans, } \frac{e_{1}}{v_{1}}=\frac{23}{205}=0, \mathrm{II} 2 & \Delta e_{1}=0, \mathrm{II} 2 \times \Delta v \\
\text { à } 5+\text { ans, } \frac{e_{2}}{v_{2}}=\frac{22,5}{225}=0, \text { IOO } & \Delta e_{2}=0, \mathrm{I} \times \Delta v
\end{array}
$$

Une étude plus précise devrait tenir compte de la réponse relative des petites tiges à la fertilisation; mais la simplification proposée ne conduit pas à de grosses erreurs, et en l'absence de données, peut être considérée comme suffisante.

\title{
I. 2. Les données économiques
}

Les valeurs présentées dans cette étude correspondent aux prix en vigueur au I novembre 1974, qui a été choisi comme date de référence.

\section{2I. Dépenses de fertilisation.}

Évaluées sur de telles bases, les dépenses occasionnées à 1'hectare par les apports PK et NPK retenus sont alors les suivants :

\author{
TABLEAU 3

\begin{tabular}{|c|c|c|c|c|}
\hline & \multicolumn{2}{|l|}{ Apport PK } & \multicolumn{2}{|l|}{ Apport $X$} \\
\hline $\begin{array}{l}\text { l'ourniture } \\
\text { d'engrais }\end{array}$ & $\begin{array}{c}150 \mathrm{~kg} \mathrm{P}_{2} \mathrm{O}_{5} \text { (superphosphate) } \\
1000 \mathrm{~kg} \mathrm{~K} \mathrm{~K}_{2} \mathrm{O}\end{array}$ & $\begin{array}{r}330 \\
50\end{array}$ & $100 \mathrm{~kg} \times$ (ammonitrate) & 230 \\
\hline Épandage & & 120 & & 45 \\
\hline Totaux .. & $\ldots \ldots \ldots \ldots \ldots \ldots \ldots \ldots \ldots \ldots$ & 500 & $\ldots \ldots+\cdots, \ldots, \ldots, \ldots$ & 275 \\
\hline
\end{tabular} \\ Détail des coûts (F/ha) \\ Details on costs of fertilization ( $\mathbf{F} /$ ha)
}

Le choix des superphosphates, en raison de leur effet plus rapide et du contingentement des scories, tend à grossir les dépenses de fertilisation.

Les charges d'épandage comprennent le coût d'incorporation des phosphates au sol nécessaire à assurer un coefficient et une vitesse d'utilisation corrects. Il n'a pas été compté dans ce coût les débroussaillements au rouleau landais préalables à l'épandage d'engrais, qui est une intervention culturale normale, à effectuer de toute façon.

La valeur d'achat de l'engrais a été légèrement majorée pour tenir compte de conditions extrêmes de livraison, qui sont en fait très rares dans les Landes. Les dépenses de fertilisation en paraissent par là même surestimées. 
I. 22. Le prix du bois sur pied.

Le prix sur pied du $\mathrm{m}^{3}$ de bois obtenu après fertilisation varie sous 1'effet combiné de plusieurs facteurs :

a) I1 augmente par paliers successifs avec la dimension des arbres abattus, 1a fertilisation qui permet une croissance plus rapide du diamètre, entraîne donc, à âge égal, une augmentation de ce prix. Mais en l'absence de lois biométriques simples et précises permettant de préjuger de l'effet de la fertilisation sur la croissance en hauteur et en section, il semble difficile d'en tenir compte.

b) La fertilisation entraîne une diminution des frais de gestion ramenés an $\mathrm{m}^{3}$ (donc du prix de revient du bois produit), qui a été négligée dans l'étude.

c) L'augmentation du volume et de l'homogénéité des lots produits entraine une diminution importante des frais d'exploitation, qui peut se traduire par une augmentation non négligeable du prix du bois sur pied, mais l'absence d'expérience en la matière rend sa mesure difficile.

d) La fertilisation modifie, encore que dans une faible mesure, les qualités physiques du bois produit, ce qui pourrait se solder par une baisse du prix du $\mathrm{m}^{3}$.

e) Enfin, faute de données sérieuses, il s'avère impossible de chiffrer l'incidence économique que pourrait avoir l'influence de la fertilisation sur l'état sanitaire des peuplements.

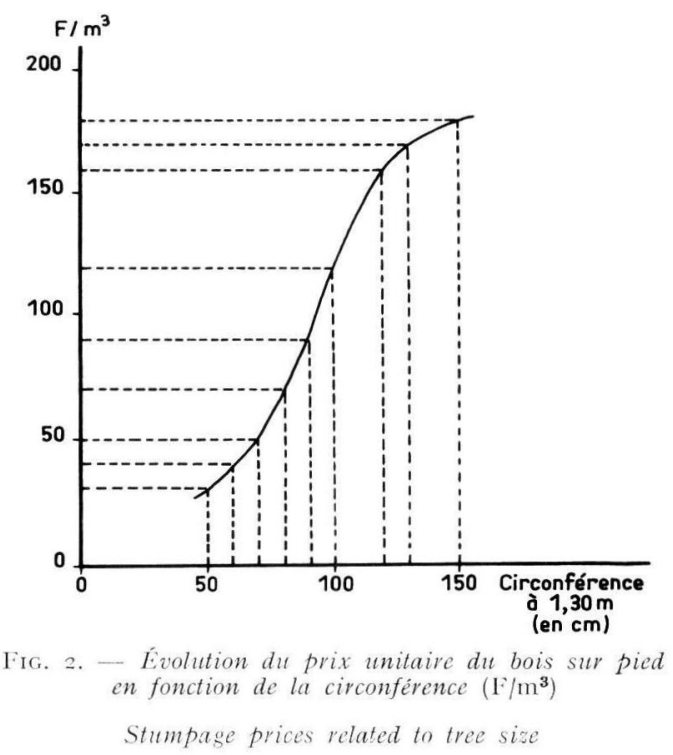

Il ressort de cette énumération que le prix du $\mathrm{m}^{3}$ de bois sur pied en peuplement fertilisé pourrait différer sensiblement du prix consenti au $\mathrm{m}^{3}$ de bois sur pied non fertilisé. Mais faute d'éléments, il est bien difficile d'en tenir compte. Tout au plus peut-on penser que ce prix ne devrait pas être inférieur à celui du bois non fertilisé. Et dans les calculs suivants, la valeur des bois fertilisés a été évaluée sur la base du prix minimum consenti pour les bois non fertilisés. Il convient de remarquer que 
cette simplification apporte un biais systématique qui tend sans doute à minimiser le résultat économique présenté.

Ces prix théoriques que présente la figure 2 ont été établis à partir des valeurs minimales en novembre $1974^{* *}$ des produits rendus bord de route en tenant compte de la répartition des bois entre les diverses utilisations.

On peut donc constater la fragilité des bases sur lesquelles ont été construits les modèles retenus. Les expériences de fertilisation de Pins maritimes adultes sont peu nombreuses, ce qui limite les possibilités d'extrapolation dans l'espace, et relativement jeunes, ce qui oblige à des extrapolations dans le temps. Pour résoudre dans une certaine mesure ces difficultés, l'étude s'est donc volontairement placée dans les conditions de coûts et de recettes les moins optimistes. Il n'en reste pas moins vrai que les valeurs qui y sont présentées sont à retenir avec une grande prudence.

\section{2. - ESSAI D’ANALYSE MICROÉCONOMIQUF, DU GAIN EN VOLUME}

On suppose que sans changer la révolution prévue, donc la date de coupe finale qui a été choisie à 60 ans, le sylviculteur recherche par l'apport d'engrais une augmentation du volume à récolter. Cette alternative, qui satisfait à un certain nombre de motivations particulières, mais qu'il ne s'agit pas de préconiser de façon systématique, constitue une première approche simple du problème : il s'agit alors de déterminer la date optimale d'application (soit le nombre d'années $n$ devant séparer l'apport phospho-potassique de la récolte ; $n=60-t)$, et la valeur du résultat économique espéré.

\section{I. - Le bénéfice apparent de l'opération fertilisation}

C'est celui qui résulte de la simple comparaison du flux des recettes réalisées et du flux des dépenses correspondantes. I1 satisfait donc aux égalités suivantes : dans lesquelles:

- pour $\mathrm{PK}, \mathrm{B}=\Delta \mathrm{P}+\Delta \mathrm{E}_{1}+\Delta \mathrm{E}_{2}-\mathrm{C}_{0}$

pour NPK, B $=\Delta \mathrm{P}+\Delta \mathrm{E}_{1}+\Delta \mathrm{E}_{2}-\left(\mathrm{C}_{0}+\mathrm{C}_{1}+\mathrm{C}_{6}\right)$
$\Delta \mathrm{P}=$ supplément de revenu qu'entraîne la fertilisation sur le gain à la récolte ;
$\Delta \mathrm{E}_{1}=$ supplément de revenu qu'entraîne la fertilisation sur le gain à l'éclaircie de $4^{6}$ ans ;
$\Delta \mathrm{E}_{2}=$ supplément de revenu qu'entraîne la fertilisation sur le gain à l'éclaircie de $5+$ ans;
$\mathrm{C}_{\mathbf{0}}=$ dépense occasionnée par l'apport $\mathrm{PK}$ à $t=0$
$\mathrm{C}_{1}=$ dépense occasionnée par l'apport $\mathrm{N}$ à $t=\mathrm{I}$
$\mathrm{C}_{\mathbf{6}}=$ dépense occasionnée par l'apport $\mathrm{N}$ à $t=6$

Mais une telle analyse est insuffisante.

\section{2. - Le bénéfice net actualisé de l'opération fertilisation (BNA)}

La fertilisation des peuplements forestiers représente un véritable investissement qui s'intègre au capital. Il faut donc comparer des flux financiers intervenant

** Les prix retenus dans le calcul présenté étant des minima, la baisse constatée lors des dernières ventes n’a qu'un faible impact sur les résultats, dont l'ordre de grandeur reste inchangé. 


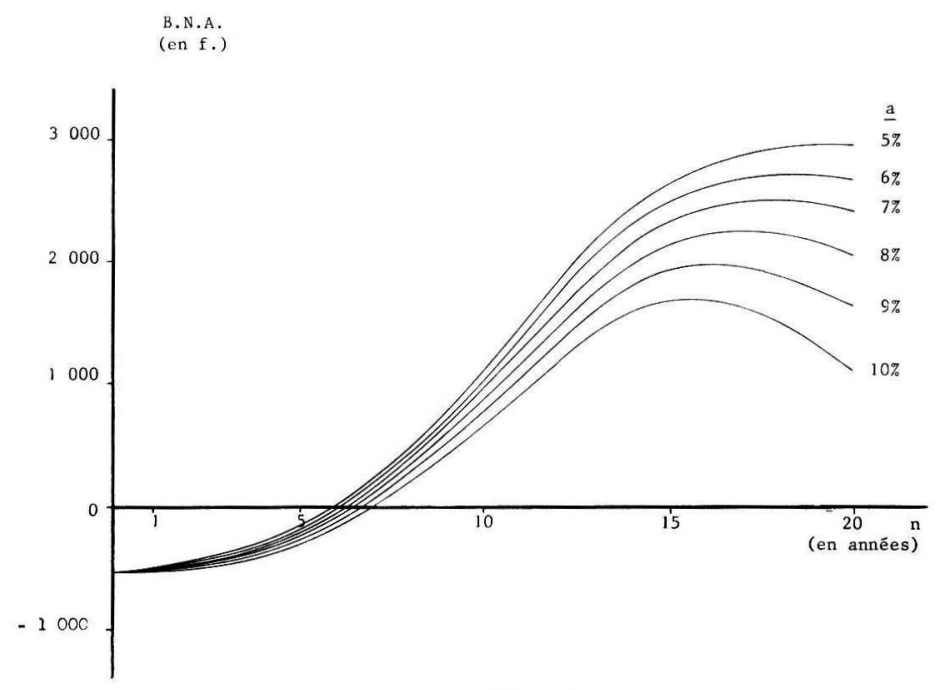

FIG. 3. - Modile PK : variation du BNA à la récolte finale (en francs) PK model: net Income Value at the final harvest cut

B.N.A.

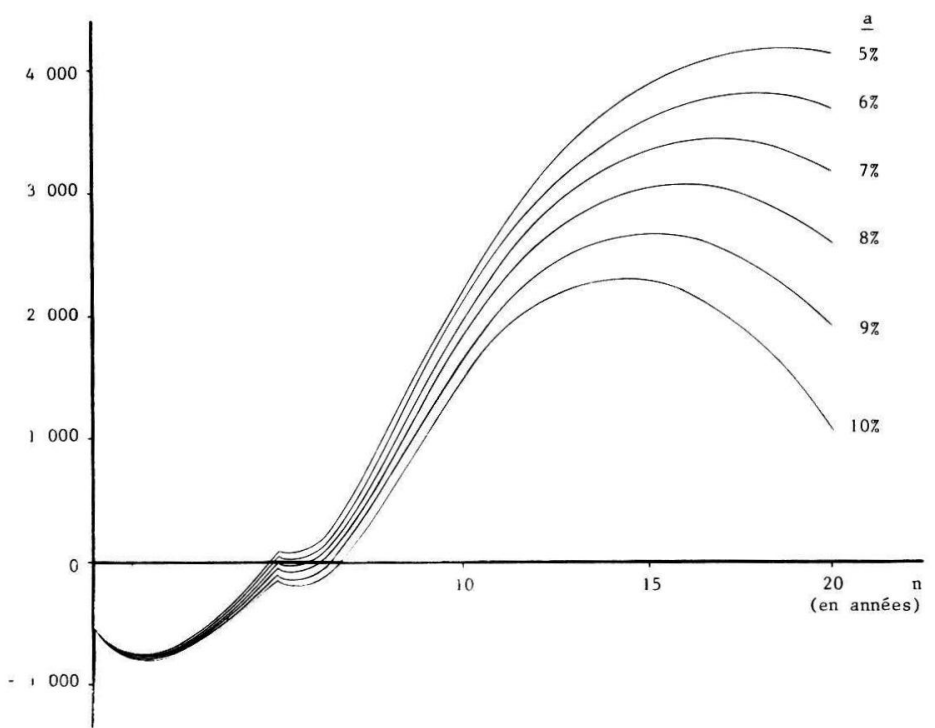

FIG. 4. - Modèle NPK : variation du BNA à la récolte finale (en francs) NPK model: net Income Value at the final harest cut 
à des dates très différentes, et le bénéfice réel que le sylviculteur est censé retirer de l'investissement varie alors avec la valeur qu'il accorde au temps. Cette valeur, essentiellement subjective, peut être matérialisée par l'artifice de calcul que représente le choix d'un taux d'actualisation $a$. Ainsi dans les conditions de l'étude :

$$
\mathrm{BNA}=f(n, a)
$$

La valeur de ce taux d'actualisation $(a)$ sera d'autant plus élevée que la préférence pour le présent sera plus forte.

Et pour un $n$ donné, à chaque valeur de $a$ choisie, correspond une valeur du bénéfice actualisé, d'autant plus forte que $a$ est faible.

Notons que lorsque $\quad a=0, \quad B N A=B$

Il a été convenu dans l'étude :

- d'actualiser sur la date de récolte (qui est fixée), ce qui présente l'inconvénient de grossir artificiellement le résultat économique.

- d'utiliser des taux d'actualisation de 5 à Io p. IOO, exprimant ainsi une préférence nette pour le présent.

Si l'apport PK a lieu $n$ années avant la récolte, ces bénéfices actualisés s'obtiennent par les formules suivantes:

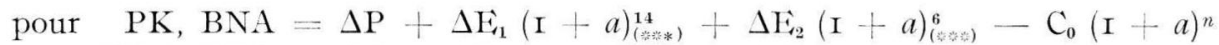

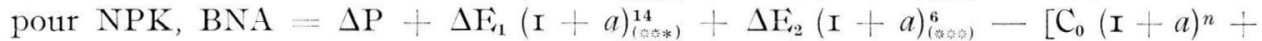

$$
\begin{aligned}
& \left.\mathrm{C}_{1}(\mathrm{I}+a)^{n-1}+\mathrm{C}_{6}(\mathrm{I}+a)^{n-6}\right]
\end{aligned}
$$

Les figures 3 et 4 résultant du calcul proposé illustrent l'évolution de ce bénéfice avec la durée d'action $n$ de l'engrais.

$$
\text { TABLEAU } 4
$$

\begin{tabular}{|c|c|c|c|}
\hline Modèle & $\begin{array}{c}a \\
(\mathrm{en} \mathrm{p} .100)\end{array}$ & $\begin{array}{l}\text { Durée d'action } \\
\text { (en années) }\end{array}$ & BNA (en F) \\
\hline \multirow{6}{*}{ PK } & j & 20 & 2945 \\
\hline & 6 & is & 2725 \\
\hline & 7 & is & 2495 \\
\hline & ২ & 17 & 2235 \\
\hline & 9 & 16 & 1965 \\
\hline & 10 & $16 i$ & 1680 \\
\hline \multirow{6}{*}{ NIK } & 5 & 19 & 4145 \\
\hline & 6 & 18 & 3805 \\
\hline & 7 & 16 & 3440 \\
\hline & 8 & 16 & 3070 \\
\hline & 9 & 15 & 2670 \\
\hline & 10 & 1't & 2270 \\
\hline
\end{tabular}

Bénéfices nets actualisés aux optima d'application

Net Income Values at optimum

\footnotetext{
*** En effet, le produit $\mathrm{E}_{1}$ de l'éclaircie de 46 ans est obtenu $60-46=\mathrm{I} 4$ ans avant la récolte finale; de même le produit $\mathrm{E}_{2}$ de l'éclaircie de 54 ans est obtenu 6 ans avant cette même récolte finale.
} 
On obtient donc pour chaque valeur de $a$ une durée d'action $n$ de l'engrais donnant le BNA maximum. L'optimum d'application de la fertilisation varie ainsi avec la valeur attribuée au temps.

\section{3. - Le tanx de rentabilité interne de l'investissement}

Un inconvénient de cette analyse réside dans le fait que le choix d'un taux d'actualisation est arbitraire, et par-là même discutable. Car chaque agent économique aura son propre taux d'actualisation, qui diminuera avec sa préférence pour le futur, donc avec sa " richesse " ou ses motivations personnelles.

Un moyen pratique de pallier cette contrainte consiste à mesurer la rentabilité de l'opération par le taux d'actualisation $r$ qui annule le BNA. Ce taux appelé taux de rentabilité interne ne dépend ni de la date choisie comme référence, ni de la valeur attribuée au temps. Le sylviculteur peut alors conclure à la rentabilité de l'opération, si $r$ est supérieur à son propre taux d'actualisation, mais une telle évaluation ne permet pas d'estimer le montant exact qu'il en retirera. On obtient $r$ en résolvant les équations: (définition des symboles p. I45.)

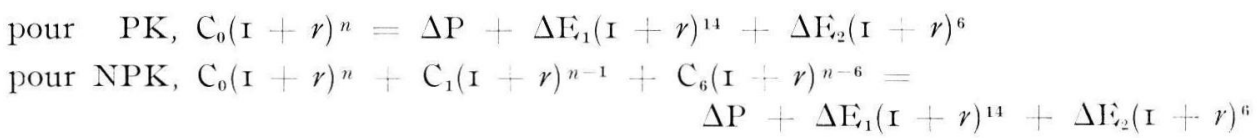

Le calcul conduit aux résultats suivants :

TABLIAL 5

Taux interne de rentabilité $\mathrm{r}$ de l'investissement

(unité $=$ p. IOO)

Internal rate of return of the investinent (r)

\begin{tabular}{|c|c|c|}
\hline$n$ & PK & NPK \\
\hline 't & négatif & négatif \\
\hline 5 & 1,3 & 6,9 \\
\hline 6; & 7,0 & $7, \ddot{2}$ \\
\hline 7 & 9,9 & $11, \ddot{2}$ \\
\hline 8 & 12,0 & 13,7 \\
\hline 9 & 13,7 & 15,5 \\
\hline 10 & 14,7 & 16,8 \\
\hline 11 & 15,3 & 16,9 \\
\hline 12 & $1.5,5$ & 16,3 \\
\hline 13 & $15, i$ & 15,0 \\
\hline $1 '$ & 15,0 & 13,8 \\
\hline 15 & $1 / 4,5$ & 12,9 \\
\hline 16 & 13,6 & 12,0 \\
\hline 17 & 12,9 & 11,5 \\
\hline 18 & 12,2 & 11,1 \\
\hline 19 & 11,7 & 10,5 \\
\hline 20 & 11,1 & 10,4 \\
\hline
\end{tabular}


$r$ varie ainsi avec $n$ en décrivant une courbe convexe vers le haut et passe par un maximum qui correspond à l'optimum théorique d'application de l'engrais, dans les conditions d'aménagement fixées. (cf. fig. 5)

Les valeurs prises par ce taux confirment la rentabilité de l'opération. Pour chaque modèle, le maximum de $r$ correspond à des durées d'action plus faibles que celles trouvées dans l'étude de l'évolution du BNA. Mais, compte tenu de l'incertitude entachant les données de base, 1'optimum résultant du calcul peut être retenu à quelques années près, surtout en ce qui concerne le modèle PK.

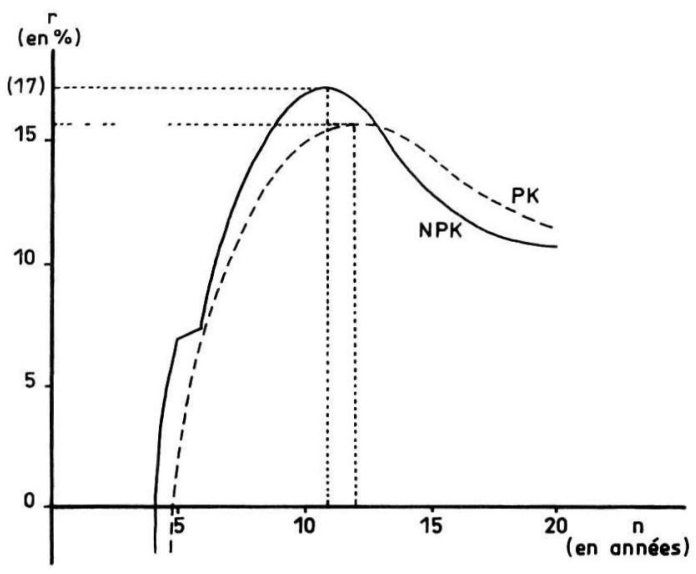

Fig. 5. - Evolution dans le temps du taux de rentabilité interne Internal rate of return related to time

On peut ainsi retenir qu'en gros, une fertilisation du type PK, apportée $\mathrm{I}_{5}$ ans environ avant la date de récolte prévue, permettrait d'obtenir un volume supplémentaire d'au moins $25 \mathrm{~m}^{3}$ par hectare et apporterait un bénéfice net actualisé au taux de 5 p. Ioo de $2500 \mathrm{~F}$. Le taux de rentabilité d'un tel investissement serait alors voisin de $I_{5}$ p. Ioo.

Il convient d'ajouter qu'une variante de la méthode du BNA consistant à comparer le prix de revient $\mathrm{du} \mathrm{m}^{3}$ supplémentaire produit par fertilisation au prix de vente de bois sur pied s'avère parfois intéressante. C'est d'ailleurs cette méthode qu'ont utilisée Bonneau M., Gelpe J. et ILLY G., dans leurs premières conclusions sur les essais de Marcheprime et de l'Hermitage. En fait, aucune des méthodes utilisées ne permet à elle seule d'illustrer de façon complète l'intérêt économique de la fertilisation, comme il en va de tout investissement forestier. La décision d'application dépend en effet de nombreuses motivations personnelles au décideur dont on ne peut plus ou moins bien rendre compte qu'en utilisant simultanément ces deux techniques de calcul. D'autre part, attribuer au temps une valeur moyenne constante sur une durée fixée est une simplification qui, dans certains cas, peut ne pas rendre compte de la réalité.

Les calculs qui précèdent se fondent sur un modèle simple, construit à partir de données certes critiquables, mais avec le souci permanent de sous-estimer le résultat économique attendu. Or les valeurs trouvées semblent intéressantes. I1 
convient toutefois de souligner que cette étude présente le résultat d'une comparaison entre certaines caractéristiques (pas toutes) d'un modèle "fertilisé ) et d'un témoin "non fertilisé " ainsi pris comme base de référence. A aucun moment du raisonnement, l'existence même du peuplement n'est remise en cause. L'étude se limite donc au cas particulier suivant : à un certain moment de la vie du peuplement, le sylviculteur apprend l'intérêt qu'il pourrait avoir à pratiquer un apport d'engrais, il cherchera à maximiser le supplément de gain qu'il peut ajouter à la solution "non fertilisée " qui aurait de toute façon été suivie si l'opportunité de la fertilisation ne s'était pas présentée.

Les solutions aux calculs précédents ne peuvent donc pas être systématiquement adoptées comme modèles de gestion; elles se contentent de donner une réponse à un problème instantané intervenant en cours de vie du peuplement. L'étude a d'ailleurs été menée sur l'intérêt que présente, à volume égal de bois produit, le raccourcissement de la révolution par apport d'engrais (BU'T'ToUD, I975).

Malgré le faible nombre de données actuellement disponibles, il semble que dans certaines conditions d'aménagement, la fertilisation des peuplements adultes de Pin maritime soit une opération intéressante dont il conviendrait d'approfondir l'étude.

La rentabilité des nouvelles techniques intensives de conduite des jeunes peuplements dans les Landes peut d'ores et déjà être considérée comme acquise. Il s'agit à présent d'effectuer dans les meilleures conditions un passage à ce système de production intensif qui permette la mise sur le marché de quantités supplémentaires de bois.

$$
\text { Reçu pour publication en mars } 19 \% 6 \text {. }
$$

\section{SUMMARY \\ ECONOMICS OF MATURE PINUS PINASTER \\ IN THE LANDES OF GASCOGNE, \\ A FIRST STUDY}

First results of experimentations settled by the National Institute for Agronomic Research lead to the conclusion that fertilizing mature stands in the Landes of Gascogne could be an interesting practice and that further study is needed.

A PK fertilization, brought almost I $_{5}$ years before the final cut, results at least in a $25 \mathrm{~m}^{3} / \mathrm{ha}$ increase of the production. The internal rate of return of such an investment would be near I 5 p. IOo.

Due to the small amount of experimental data, forecasting the influence of late fertilization is hardly possible. However, further technical investigations seem to be badly needed.

\section{RÉFÉRENCES BIBLIOGRAPHIQUES}

Bonnead M., Gelpe J., Ilify, G., I972. "Résultats de deux essais de fertilisation sur Pin maritime adulte dans les Landes." Ann. Sci. Forest., I.N.R.A., 29 (3), 353-367.

Centre national de recherches forestières, i973. Tables de production pour les forêts françaises. ENGREF Nancy, 33-35.

Buttoud G., 1975. L'économie de la fertitisation du Pin maritime adulte dans les Landes de Gascogne. Laboratoire d'Économie forestière. Nancy, 74 p. 